\title{
Association of lymphocyte count and incidence of maternal fever in epidural analgesia-involved labor
}

\author{
Jing Chai ${ }^{1,2 \#}$, Lijie Jia ${ }^{1,2 \#}$, Huimin Cao ${ }^{1,2}$, Xiaoyu Zhang ${ }^{1,2}$, Zifeng $\mathrm{Xu}^{1,2}$ \\ ${ }^{1}$ Department of Anesthesiology, International Peace Maternity and Child Health Hospital, Shanghai Jiao Tong University School of Medicine, \\ Shanghai, China; ${ }^{2}$ Shanghai Key Laboratory of Embryo Original Diseases, Shanghai, China \\ Contributions: (I) Conception and design: J Chai, L Jia, Z Xu; (II) Administrative support: Z Xu; (III) Provision of study materials or patients: J Chai, \\ L Jia, H Cao; (IV) Collection and assembly of data: J Chai, L Jia, H Cao, X Zhang; (V) Data analysis and interpretation: J Chai, L Jia, Z Xu; (VI) \\ Manuscript writing: All authors; (VII) Final approval of manuscript: All authors. \\ "These authors contributed equally to this work. \\ Correspondence to: Zifeng Xu. Department of Anesthesiology, International Peace Maternity and Child Health Hospital, Shanghai Jiao Tong \\ University School of Medicine, 910 Hengshan Road, Shanghai, China. Email: xuzf@shsmu.edu.cn.
}

Background: Evidence showed that the use of epidural analgesia increased the risk of maternal fever during labor, and the potential mechanisms involved inflammation. Lymphocyte played a role in the inflammatory responses, and lymphopenia predicted adverse outcomes for patients. The aim of this study was to investigate whether lymphocyte count was associated with the incidence of intrapartum fever in nulliparous women undergoing vaginal delivery.

Methods: In this retrospective cohort study, electronic medical record data at the International Peace Maternity and Child Health Hospital (IPMCH, Shanghai, China) between 2012 and 2017 were extracted. The primary outcome of this study was baseline lymphocyte count in febrile and afebrile parturients who received labor epidural analgesia. Other confounding factors associated with the development of intrapartum fever were analyzed using multivariable logistic regression.

Results: The final study population consisted of 36,562 parturients, of whom 18,952 (51.8\%) received labor epidural analgesia. Labor epidural analgesia was associated with an increased risk of maternal fever [relative risk: 4.55; 95\% confidence interval (CI): 4.16-4.98; $\mathrm{P}<0.001$ ]. Among the parturients who received epidural analgesia, both baseline lymphocyte count and lymphocyte percentage were lower in the febrile patients than those in the afebrile patients $(\mathrm{P}<0.001 ; \mathrm{P}=0.015)$. The lower $\left(<1.1 \times 10^{9} / \mathrm{L}\right)$ baseline lymphocyte was associated with a higher risk of maternal fever (adjusted odds ratio: 1.30, 95\% CI: $1.14-1.48 ; \mathrm{P}<0.001$ ).

Conclusions: Our results suggested that lower lymphocyte baseline indicated an increased risk of maternal fever during epidural analgesia-involved labor. Further studies are needed to confirm the findings and determine the potential mechanisms.

Keywords: Epidural analgesia; labor; lymphocyte; maternal fever

Submitted Feb 19, 2020. Accepted for publication Sep 25, 2020.

doi: $10.21037 /$ atm-20-1724

View this article at: http://dx.doi.org/10.21037/atm-20-1724

\section{Introduction}

Intrapartum fever occurred in approximately onefourth of all labors $(1,2)$. Maternal fever was associated with more frequent obstetric interventions, unnecessary administration of antibiotics, a higher rate of cesarean section, and adverse neonatal outcomes $(3,4)$. Intrapartum fever was first proposed to be related to epidural analgesia in 1989 in Lancet (5). A following retrospective "beforeand-after" study strengthened the validity of this association and demonstrated that labor epidural analgesia increased the incidence of intrapartum fever (6). Recently, epidural 
analgesia-involved labor becomes popular in developing regions. However, the factors that cause epidural analgesiarelated fever and their potential mechanisms remained unclear.

The current evidence demonstrated that most intrapartum fever did not have infectious etiologies, but involved the inflammatory response and immune modulation (7). The baseline interleukin-6 (IL-6) was associated with maternal intrapartum fever (8), however, it is not feasible to measure cytokine levels in clinical practice. Lymphocyte is the essential part of the cellular immune response. The decreased lymphocyte count predicted adverse outcomes in non-obstetric patients (9-11). However, the association between lymphocyte count and maternal fever has not been assessed. This retrospective cohort study aimed to investigate whether low lymphocyte baseline was associated with the incidence of intrapartum fever in a large cohort of full-term nulliparous women undergoing vaginal delivery. The findings of this study will assist both obstetricians and anesthesiologists in estimating the potential risk of intrapartum fever before the administration of labor epidural analgesia. We present the following article in accordance with the STROBE reporting checklist (available at http://dx.doi.org/10.21037/atm-20-1724).

\section{Methods}

The study was conducted in accordance with the Declaration of Helsinki (as revised in 2013). The study was approved by the Ethics Committee of the International Peace Maternity and Child Health Hospital (IPMCH, No. GKLW2017-153), and the protocol was registered at http://www.chictr.org.cn (ChiCTR1900027694). Individual consent for this retrospective analysis was waived. Electronic medical record data of deliveries at IPMCH between January 1, 2012 and December 31, 2017 were extracted by investigators. The inclusion criterion was fullterm vaginal delivery with live birth. Parturients who were parous, or who had multiple gestations were excluded. In addition, some parturients had no records of the white blood cells (WBCs) within 24 hours before delivery; these parturients were also excluded for further analyses.

Blood samples were taken from the median cubital vein for routine blood test when the parturients arrived at the labor and delivery unit. When a cervical dilatation of $3.0 \mathrm{~cm}$ or greater was reached, the epidural analgesia was administrated to parturients who requested for epidural analgesia and did not have the epidural contraindications (including coagulation dysfunction and lumbar surgery history). Baseline temperatures were recorded when parturients arrived at labor and delivery unit. Temperatures were assessed every 2 hours by the nursing staff during labor and delivery. If a temperature of $37.5^{\circ} \mathrm{C}$ was reached, the diagnosis of fever was made by the obstetric provider, temperatures were then assessed hourly. Following the diagnosis of fever, the antibiotics was administrated based on the patient's clinical presentation and blood test results. The duration of labor was limited to 24 hours according to the IPMCH rules.

The extracted data included age, body mass index (BMI), gestational age, parity, cervical examinations, labor length (the time from regular uterine contraction until placental expulsion), birth weight, and WBC test results. Parturients with intrapartum fever had several blood tests during labor; the results of the first blood test taken within 24 hours before delivery were extracted for analyses. More than 3,000 records were randomly selected for manual evaluation to confirm the accuracy of the data.

\section{Statistical analysis}

The primary outcome was baseline lymphocyte count, defined as the lymphocyte count recorded in the first blood routine test within 24 hours before delivery, usually at the onset of labor or very close to the onset of labor. The normal distribution of continuous variables was determined using the Shapiro-Wilk test. Continuous variables were presented as mean \pm standard deviation (SD), or as median [interquartile range (IQR)]. Counting variables were presented as numbers (percentage). For variables with missing data (maternal age missing in $1.8 \%$, and BMI missing in $2.5 \%$ ), multiple imputation according to the Markov chain Monte Carlo method was used by creating five imputed data sets for analyses. Quantitive data were compared either by independent $t$-test for normally distributed data (including age, BMI, labor length, and birth weight), or Mann-Whitney test for the non-normally distributed data (including WBC count, neutrophil count, neutrophil percentage, monocyte count, monocyte percentage, lymphocyte count, lymphocyte percentage, and lymphocyte to neutrophil ratio). Counting data were compared using the chi-squared test. The relationships between baseline lymphocyte count and the patient characteristic data with maternal fever were first analyzed by individual logistic regression analysis to identify variables associated with the incidence of maternal 


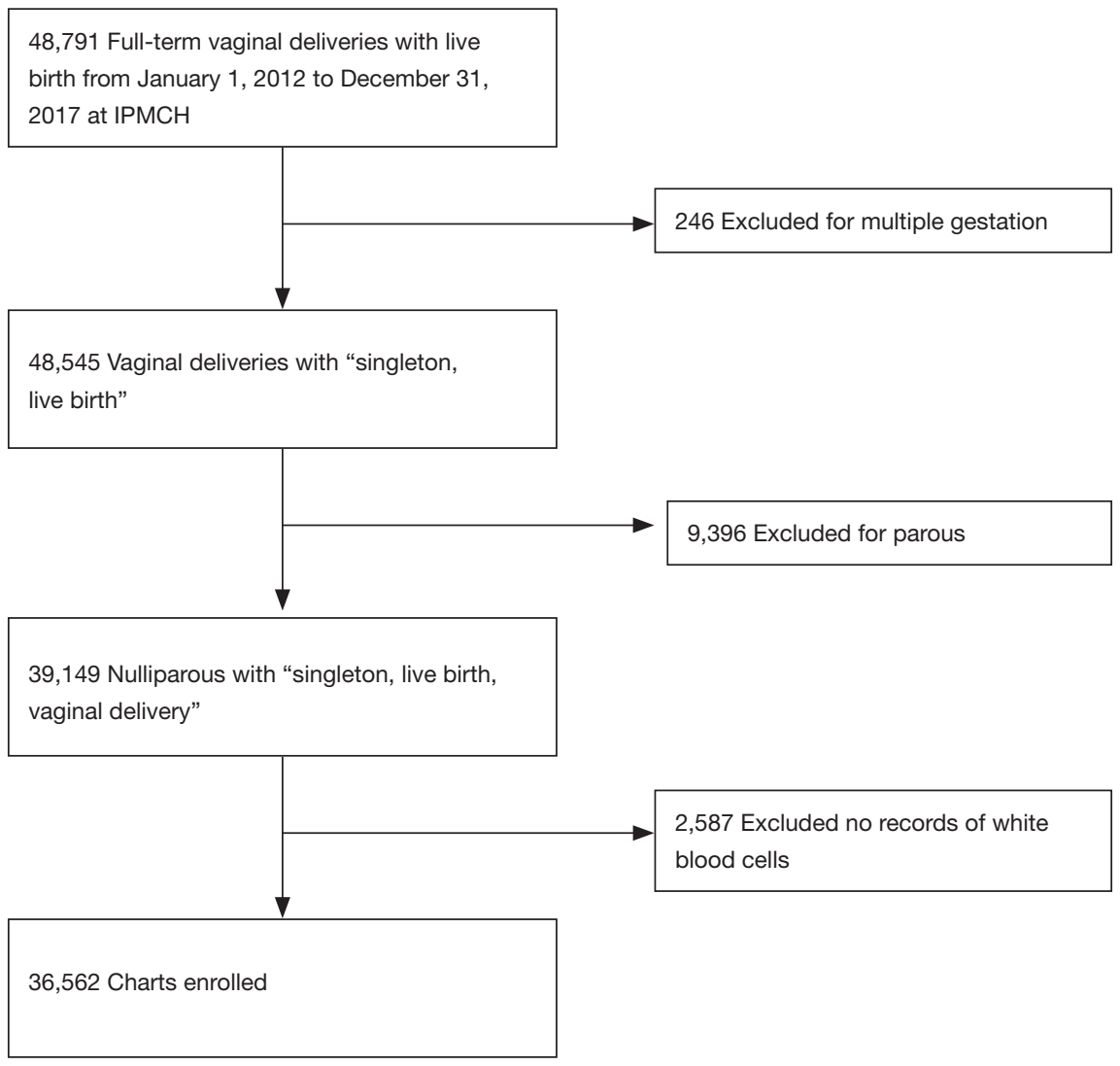

Figure 1 Participant flowchart. IPMCH, International Peace Maternity and Child Health Hospital.

fever. Multivariable forward selection logistic regression analysis was then used to identify variables independently associated with outcomes at $\mathrm{P}<0.05$. All statistical analyses were performed using Statistical Package of Social Sciences version 25.0 for Macintosh (IBM Corp., USA).

\section{Results}

A total of 48,791 parturients met the inclusion criterion. From this initial cohort, 246 cases were excluded due to multiple gestations, 9,396 were excluded due to parity, and 2,587 were excluded because they did not have records of WBC (Figure 1). The final study population consisted of 36,562 parturients, of whom 3,268 (8.9\%) were recorded as having a fever during labor. The epidural analgesia was performed to 18,952 parturients (51.8\%). Parturients receiving epidural analgesia were more likely to develop fever during labor: $14.3 \%(2,714 / 18,952)$ in epiduralanalgesia group compared with $3.1 \%(554 / 17,610)$ in no epidural group [relative risk: $4.55 ; 95 \%$ confidence interval (CI): 4.16-4.98; $\mathrm{P}<0.001$; Table S1], suggesting an increased risk of intrapartum fever among parturients who received epidural analgesia.

The parturients were grouped according to epidural analgesia administration and fever status (febrile or afebrile). In the parturients without epidural analgesia, there was no difference in baseline WBC count, neutrophil count, neutrophil percentage, lymphocyte count, lymphocyte percentage, or the lymphocyte to neutrophil ratio between febrile and afebrile women. Baseline monocyte count of febrile patients was higher than that of afebrile patients $(\mathrm{P}=0.039$; Table 1). However, there was no difference in baseline monocyte percentage between febrile and afebrile women who did not receive epidural analgesia. Among parturients administrated with epidural analgesia, both baseline lymphocyte count (febrile patients: $1.53 / \mathrm{nL}$; afebrile patients: $1.57 / \mathrm{nL} ; \mathrm{P}<0.001)$ and lymphocyte percentage (febrile patients: $18.4 \%$; afebrile patients: $18.7 \% ; \mathrm{P}=0.015)$ were significantly lower in febrile patients than in afebrile patients (Table 1). Baseline WBC count, neutrophil count, neutrophil percentage, or monocyte count was not different between febrile 
Table 1 WBC baseline according to epidural analgesia administration and fever status

\begin{tabular}{|c|c|c|c|c|c|c|}
\hline Variables & \multicolumn{3}{|c|}{ No epidural analgesia } & \multicolumn{3}{|c|}{ Epidural analgesia } \\
\hline WBC $\left(10^{9} / \mathrm{L}\right)$ & $8.6(7.3-10.2)$ & $8.6(7.4-10.3)$ & 0.749 & $8.6(7.3-10.0)$ & $8.5(7.1-10.0)$ & 0.106 \\
\hline Neutrophil (10\%/L) & $6.28(5.08-7.76)$ & $6.21(5.14-7.79)$ & 0.750 & $6.21(5.04-7.60)$ & $6.16(5.02-7.59)$ & 0.332 \\
\hline Neutrophil (\%) & $73.5(68.5-77.9)$ & $73.4(68.7-78.6)$ & 0.388 & $73.3(68.6-77.6)$ & $73.3(68.9-77.7)$ & 0.226 \\
\hline Monocyte (\%) & $6.8(5.6-8.1)$ & $6.9(5.8-8.2)$ & 0.107 & $6.8(5.7-8.1)$ & $7.0(5.8-8.2)$ & 0.007 \\
\hline Lymphocyte $\left(10^{9} / \mathrm{L}\right)$ & $1.57(1.30-1.89)$ & 1.54 (1.28-1.87) & 0.118 & $1.57(1.31-1.88)$ & $1.53(1.27-1.83)$ & $<0.001$ \\
\hline Lymphocyte (\%) & $18.6(14.8-22.8)$ & $18.2(14.1-22.6)$ & 0.105 & $18.7(15.1-22.7)$ & $18.4(14.8-22.5)$ & 0.015 \\
\hline Lymphocyte/neutrophil & $0.25(0.19-0.33)$ & $0.25(0.18-0.33)$ & 0.144 & $0.26(0.20-0.33)$ & $0.25(0.19-0.33)$ & 0.028 \\
\hline
\end{tabular}

Variables were presented as median (IQR). WBC, white blood cell; IQR, interquartile range.

Table 2 Characteristics of study population according to epidural analgesia administration and fever status

\begin{tabular}{|c|c|c|c|c|c|c|}
\hline Variables & \multicolumn{3}{|c|}{ No epidural analgesia } & \multicolumn{3}{|c|}{ Epidural analgesia } \\
\hline Maternal age (year) & $29.3 \pm 3.0$ & $29.4 \pm 2.9$ & & $29.5 \pm 3.0$ & $29.8 \pm 3.1$ & \\
\hline Maternal age $\geq 35$ years & $864(5.1)$ & $27(4.9)$ & 0.911 & $973(6.0)$ & $192(7.1)$ & 0.033 \\
\hline BMI $\left(\mathrm{kg} / \mathrm{m}^{2}\right)$ & $25.9 \pm 2.7$ & $26.1 \pm 2.8$ & & $26.0 \pm 2.7$ & $26.3 \pm 2.7$ & \\
\hline Labor length (min) & $351.7 \pm 158.2$ & $405.4 \pm 185.8$ & & $498.6 \pm 197.3$ & $586.5 \pm 198.7$ & \\
\hline Labor length $>600 \mathrm{~min}$ & $1,349(7.9)$ & $89(16.1)$ & $<0.001$ & $4,236(26.1)$ & $1,139(42.0)$ & $<0.001$ \\
\hline Birth weight (kg) & $3.3 \pm 0.4$ & $3.3 \pm 0.4$ & & $3.4 \pm 0.4$ & $3.4 \pm 0.3$ & \\
\hline Birth weight $>4.0 \mathrm{~kg}$ & $447(2.6)$ & $17(3.1)$ & 0.499 & $627(3.9)$ & $112(4.1)$ & 0.523 \\
\hline
\end{tabular}

Continuous variables were presented as mean $\pm \mathrm{SD}$, counting variables were presented as number (percentage). BMI, body mass index; $\mathrm{SD}$, standard deviation.

and afebrile patients receiving epidural analgesia. The monocyte percentage (febrile patients: $7.0 \%$; afebrile patients: $6.8 \% ; \mathrm{P}=0.007)$ and lymphocyte to neutrophil ratio (febrile patients: 0.25 ; afebrile patients: $0.26 ; \mathrm{P}=0.028$ ) were significantly different between febrile and afebrile parturients in epidural group (Table 1).

The characteristics of parturients stratified by epidural analgesia administration and fever status (febrile or afebrile) are shown in Table 2. In the non-epidural group, labor duration was longer in febrile parturients (febrile patients: $405.4 \pm 185.8$ minutes; afebrile patients: $351.7 \pm$ 158.2 minutes), and intrapartum fever occurred more often in the women who had a longer duration of labor
$(\mathrm{P}<0.001 ;$ Table 2). There was no significant difference between maternal age, BMI, birth weight, or lymphocyte count between febrile and afebrile patients in the nonepidural group. In the epidural analgesia group, age (febrile patients: $29.8 \pm 3.1$ years; afebrile patients: $29.5 \pm 3.0$ years; $\mathrm{P}=0.033$ ) and BMI (febrile patients: $26.3 \pm 2.7 \mathrm{~kg} / \mathrm{m}^{2}$; afebrile patients: $\left.26.0 \pm 2.7 \mathrm{~kg} / \mathrm{m}^{2} ; \mathrm{P}<0.001\right)$ were greater in the febrile group; patients who developed intrapartum fever were more likely to be older and obese (Table 2). The labor duration of febrile parturients was significantly longer than that of afebrile parturients in the epidural analgesia group (febrile patients: $586.5 \pm 198.7$ minutes; afebrile patients: 498.6 \pm 197.3 minutes; $\mathrm{P}<0.001$; Table 2). There was no 
Table 3 Characteristics associated with maternal fever in epidural-associated labor

\begin{tabular}{lcc}
\hline Variables & Crude odds ratio (95\% Cl) & Adjusted odds ratio (95\% Cl) \\
\hline Maternal age $\geq 35$ years & $1.19(1.02-1.40)$ & $1.01(0.89-1.02)$ \\
$\mathrm{BMI} \geq 25 \mathrm{~kg} / \mathrm{m}^{2}$ & $1.27(1.16-1.39)$ & $1.23(1.13-1.35)^{\star}$ \\
Lymphocyte baseline $<1.1 \times 10^{9} / \mathrm{L}$ & $1.28(1.12-1.46)$ & $1.30(1.14-1.48)^{\star}$ \\
Labor length $>600 \mathrm{~min}$ & $2.05(1.88-2.23)$ & $2.03(1.87-2.21)^{\star}$ \\
\hline
\end{tabular}

*, $\mathrm{P}<0.05$. $\mathrm{Cl}$, confidence interval; $\mathrm{BMI}$, body mass index.

significant difference in birth weight between parturients with intrapartum fever and parturients without fever. In our hospital, the normal lymphocyte count range is $1.11-3.2 \times 10^{9} / \mathrm{L}$. Records of baseline lymphocyte counts showed that parturients with intrapartum fever were more likely to have lower baseline lymphocyte counts $\left(<1.1 \times 10^{9} / \mathrm{L}\right.$; $\mathrm{P}<0.001$; Table 2).

Based on the results shown in Table 2, we further constructed a logistic regression model to evaluate risk factors of intrapartum fever in epidural-involved labor. Maternal age, BMI, lymphocyte baseline, and labor duration were selected in the model. Following multivariable logistic regression, it was found that greater BMI, longer duration of labor, and lower lymphocyte baseline were independently associated with the incidence of intrapartum fever. The first two have been reported in previous studies (12); it was highlighted that the lower $\left(<1.1 \times 10^{9} / \mathrm{L}\right)$ baseline lymphocyte was associated with a higher risk of fever during labor (adjusted odds ratio: 1.30, 95\% CI: $1.14-1.48$; $\mathrm{P}<0.001$; Table 3) in the parturients receiving epidural analgesia.

Finally, the demographics of parturients stratified by administration of epidural analgesia were described in Table S1. The elder ( $\geq 35$ years) parturients were more likely to request for epidural analgesia. The labor duration of epidural analgesia group was significantly longer than that of the non-epidural group, and the birth weight was greater in the epidural group compared with the nonepidural group. The percentage of parturients with lower $\left(<1.1 \times 10^{9} / \mathrm{L}\right)$ baseline lymphocyte was not significantly different between the epidural group and the non-epidural group.

\section{Discussion}

The main finding of this study was that lower lymphocyte baseline is associated with an increased incidence of maternal fever in the epidural analgesia-involved labor. Our result was consistent with the well-established understanding that the link between epidural analgesia and maternal fever in labor likely involves non-infectious inflammation $(1,4,13)$. While labor analgesia has become increasingly prevalent throughout developing regions in recent years, some parturients prefer natural labor without pain-relief interventions. In IPMCH, a large community hospital in Shanghai, the rate of epidural analgesia-involved delivery was approximately $50 \%$, which provided the ideal populations for clinical investigation. Previous studies showed that cytokines baseline was associated with maternal intrapartum fever (8). However, our study indicated that lymphocyte count, which is easier to assess clinically, is also associated with intrapartum fever.

Since the first report of epidural-related intrapartum fever 30 years ago (5), the maternal fever during labor and the potential link with epidural analgesia have received widespread attention, but not yet been intensively investigated. A review consisting 22 studies demonstrated that the intrapartum fever occurred in approximately $20 \%$ of laboring women with epidural analgesia, which was slightly higher than the febrile incidence in our study (1). A possible reason for this could be that only vaginal deliveries were included in this study. Some febrile parturients turned to non-elective cesarean section when the fetal heart rate was compromised or parturients presented severe infection. Another reason could be the time at which the epidurals were placed at IPMCH. In our study, the epidural analgesia was administrated to parturients when a cervical dilatation of $3.0 \mathrm{~cm}$ or greater was reached. This protocol shortened the length of exposure to epidural analgesia (14) compared with the early epidural labor analgesia, and might reduce the rate of intrapartum fever (12). Sharpe et al. recommended delaying epidural labor analgesia for a subset of women "primed" to develop epidural fever (4). Our findings of this study might provide evidence for identifying this subset of parturients.

The mechanism by which epidural analgesia played in maternal fever was unclear $(15,16)$. The failure of 
prophylactic antibiotic therapy and the effectiveness of glucocorticoids implied a noninfectious etiology (17-19). Several studies that investigated pro-inflammatory cytokines, such as IL-6, found elevated levels of IL-6 in febrile parturients $(20,21)$. The higher IL-6 baseline on admission was showed to be related to an increased risk of developing intrapartum fever (8). This suggested that the inflammatory state of parturient potentially provided the fever foundation, and epidural analgesia might act as an accelerator. A recent study examined the immunomodulation of mononuclear leucocytes by bupivacaine (13). Although it was unclear whether bupivacaine or other local anesthetics caused direct injury to immune cells (22), the researchers proposed that mononuclear leucocytes and their immunomodulation are involved in fever development. Lymphocyte decrease is an unfavorable prognostic factor for critical patients (9-11). In the non-obstetric patients, possible causes of the low lymphocyte levels included the impairment of microcirculations, and increased corticosteroid hormones, cortisol, and catecholamine in response to a pathologic process $(23,24)$. The mechanism of lymphocyte count reduction in parturients closing to give birth remains elusive, however, similar to previous studies, our study provided primary evidence that lower lymphocyte count can be an indicator of an inflammatory state, which might pre-dispose parturients to fever, particularly for women who requested for epidural analgesia. In our study, febrile parturients without receiving epidural analgesia also had lower lymphocyte baseline compared with afebrile parturients, but the difference was not statistically significant. This might be attributed to the small sample size of febrile parturients in the non-epidural group. Although this study cannot determine the causative role of lymphocytes in the development of epidural analgesiainvolved maternal fever, and although women without epidural analgesia or with a normal lymphocyte count can still developed fever, the current findings suggested that parturients with lower lymphocyte baseline might have different immune reaction to epidural analgesia during labor, increasing the risk of fever.

Similarly to temperature fluctuations that occurred in menstrual cycle, it is physiologic that body temperature increases approximately 0.2 of a degree over 10 hours of labor due to the effects of estrogen and progesterone $(12,25,26)$. Intrapartum fever, however, was unlikely to present as normal temperature variation and the potential mechanisms would be different from that occurred in the menstrual cycle. Inflammation was stimulated since the onset of labor and changed over time during active labor (27). The factors affecting intrapartum maternal temperature have been investigated for years. Besides epidural analgesia, the positive factors reported to affect maternal temperature included nulliparity, long labor duration, high BMI, chorioamnionitis, greater oxytocin doses, and lower magnesium doses $(12,28,29)$. The pain during labor stimulated endogenous production of steroid; while epidural analgesia reduced the pain-stress responses, the pre-existing inflammation therefore easily lead to fever (4). Our findings confirmed the previously inflammationrelated etiology and suggested epidural-related fever likely had multiple causes, consisting maternal baseline inflammatory state and the immune reactions throughout the labor process. One important point should be noticed is that the specific inflammatory components of labor may be necessary for the development of intrapartum fever, because the same results were not found in the controlled parturients undergoing elective cesarean section $(4,13)$.

There were several limitations in the study. First, in this retrospective study, the temperature of parturients was recorded every 2 hours during labor; we were unable to get the highest temperature or assess their febrile degree. As a result, only the presence of fever, measured by "yes or no", was used for analyses in the study. Given this, we were unable to determine any link between the degree of reduction in lymphocyte baseline and the febrile degree. Additionally, while data from 2012 to 2017 were obtained from electric medical records, temperature measurement protocol changed from oral temperature to tympanic temperature after 2015. We could not, therefore, determine the accuracy of temperature measurement devices. Systematic bias, however, could be balanced between groups. Secondly, the baseline lymphocyte counts analyzed in this study was not performed at a definitely set time point; it was at the onset of labor or very near to the onset of labor. Because the onset of labor was not usually determined in advance, even in the prospective study, lymphocyte could not be measured in a restricted interval around admission to labor. Thirdly, we did not investigate the changes in lymphocyte levels during labor and delivery. Increasing leukocyte counts appeared to be linearly related to the duration of elapsed labor (30). Blood test was not usually performed after delivery for most parturients, thus the changes of lymphocyte count during labor would be investigated in further prospective studies. In addition, our findings cannot be extended to parous women, because they 
have a much shorter labor duration, and the incidence of intrapartum fever was significantly lower in parous women than nulliparous women (28).

In conclusion, our results suggest that lower lymphocyte baseline indicated an increased risk of developing fever during epidural analgesia-involved labor. Further prospective studies are required to confirm the findings and determine the potential mechanisms.

\section{Acknowledgments}

We gratefully acknowledge the contributions and efforts of doctors, nurses and technicians involved in data collection and extraction for this study. The authors also thank Dr. Shan Luo (from Department of Mathematics, Shanghai Jiao Tong University, Shanghai, China) for the support of statistical analysis.

Funding: This work was supported by the Clinical Science and Technology Innovation Program from the Shanghai Shen Kang Hospital Development Center (grant number SHDC12016225), and the Interdisciplinary Program of Shanghai Jiao Tong University (grant number ZH2018QNA33).

\section{Footnote}

Reporting Checklist: The authors have completed the STROBE reporting checklist. Available at http://dx.doi. org/10.21037/atm-20-1724

Data Sharing Statement: Available at http://dx.doi. org/10.21037/atm-20-1724

Peer Review File: Available at http://dx.doi.org/10.21037/ atm-20-1724

Conflicts of Interest: All authors have completed the ICMJE uniform disclosure form (available at http://dx.doi. org/10.21037/atm-20-1724). The authors have no conflicts of interest to declare.

Ethical Statement: The authors are accountable for all aspects of the work in ensuring that questions related to the accuracy or integrity of any part of the work are appropriately investigated and resolved. The study was conducted in accordance with the Declaration of Helsinki (as revised in 2013). The study was approved by the Ethics Committee of the International Peace Maternity and Child Health Hospital (IPMCH, No. GKLW2017153), and the protocol was registered at http://www.chictr. org.cn (ChiCTR1900027694). Individual consent for this retrospective analysis was waived.

Open Access Statement: This is an Open Access article distributed in accordance with the Creative Commons Attribution-NonCommercial-NoDerivs 4.0 International License (CC BY-NC-ND 4.0), which permits the noncommercial replication and distribution of the article with the strict proviso that no changes or edits are made and the original work is properly cited (including links to both the formal publication through the relevant DOI and the license). See: https://creativecommons.org/licenses/by-nc-nd/4.0/.

\section{References}

1. Sultan P, David AL, Fernando R, et al. Inflammation and epidural-related maternal fever: proposed mechanisms. Anesth Analg 2016;122:1546-53.

2. Segal S. Labor epidural analgesia and maternal fever. Anesth Analg 2010;111:1467-75.

3. Yin H, Hu R. A cohort study of the impact of epidural analgesia on maternal and neonatal outcomes. J Obstet Gynaecol Res 2019;45:1435-41.

4. Sharpe EE, Arendt KW. Epidural labor analgesia and maternal fever. Clin Obstet Gynecol 2017;60:365-74.

5. Fusi L, Steer PJ, Maresh MJ, et al. Maternal pyrexia associated with the use of epidural analgesia in labour. Lancet 1989;1:1250-2.

6. Yancey MK, Zhang J, Schwarz J, et al. Labor epidural analgesia and intrapartum maternal hyperthermia. Obstet Gynecol 2001;98:763-70.

7. Eltzschig HK, Lieberman ES, Camann WR. Regional anesthesia and analgesia for labor and delivery. N Engl J Med 2003;348:319-32.

8. Riley LE, Celi AC, Onderdonk AB, et al. Association of epidural-related fever and noninfectious inflammation in term labor. Obstet Gynecol 2011;117:588-95.

9. Warny M, Helby J, Nordestgaard BG, et al. Lymphopenia and risk of infection and infection-related death in 98,344 individuals from a prospective Danish population-based study. PLoS Med 2018;15:e1002685.

10. Pontiroli AE, Loreggian L, Rovati MPL, et al. Length of hospitalization is associated with selected biomarkers (albumin and lymphocytes) and with co-morbidities: study on 4000 patients. Biomark Res 2017;5:13.

11. Edwards MR, Sultan P, del Arroyo AG, et al. Metabolic 
dysfunction in lymphocytes promotes postoperative morbidity. Clin Sci (Lond) 2015;129:423-37.

12. Frölich MA, Esame A, Zhang K, et al. What factors affect intrapartum maternal temperature? A prospective cohort study: maternal intrapartum temperature. Anesthesiology 2012;117:302-8.

13. Del Arroyo AG, Sanchez J, Patel S, et al. Role of leucocyte caspase-1 activity in epidural-related maternal fever: a single-centre, observational, mechanistic cohort study. Br J Anaesth 2019;122:92-102.

14. Wong CA, Scavone BM, Peaceman AM, et al. The risk of cesarean delivery with neuraxial analgesia given early versus late in labor. N Engl J Med 2005;352:655-65.

15. Goetzl L. Epidural analgesia and maternal fever: a clinical and research update. Curr Opin Anaesthesiol 2012;25:292-9.

16. Goetzl L. Epidural fever in obstetric patients: it's a hot topic. Anesth Analg 2014;118:494-5.

17. Sharma SK, Rogers BB, Alexander JM, et al. A randomized trial of the effects of antibiotic prophylaxis on epiduralrelated fever in labor. Anesth Analg 2014;118:604-10.

18. Goetzl L, Zighelboim I, Badell M, et al. Maternal corticosteroids to prevent intrauterine exposure to hyperthermia and inflammation: a randomized, doubleblind, placebo-controlled trial. Am J Obstet Gynecol 2006;195:1031-7.

19. Goodier C, Newman R, Hebbar L, et al. Maternal epidural steroids to prevent neonatal exposure to hyperthermia and inflammation. Am J Perinatol 2019;36:828-34.

20. Goetzl L, Evans T, Rivers J, et al. Elevated maternal and fetal serum interleukin-6 levels are associated with epidural fever. Am J Obstet Gynecol 2002;187:834-8.

Cite this article as: Chai J, Jia L, Cao H, Zhang X, Xu Z. Association of lymphocyte count and incidence of maternal fever in epidural analgesia-involved labor. Ann Transl Med 2020;8(23):1584. doi: 10.21037/atm-20-1724
21. Smulian JC, Bhandari V, Vintzileos AM, et al. Intrapartum fever at term: serum and histologic markers of inflammation. Am J Obstet Gynecol 2003;188:269-74.

22. Yue HL, Shao LJ, Li J, et al. Effect of epidural analgesia with $0.075 \%$ ropivacaine versus $0.1 \%$ ropivacaine on the maternal temperature during labor: a randomized controlled study. Chin Med J (Engl) 2013;126:4301-5.

23. Bergquist J, Tarkowski A, Ewing A, et al. Catecholaminergic suppression of immunocompetent cells. Immunol Today 1998;19:562-7.

24. Lomivorotov VV, Efremov SM, Boboshko VA, et al. Preoperative total lymphocyte count in peripheral blood as a predictor of poor outcome in adult cardiac surgery. $\mathrm{J}$ Cardiothorac Vasc Anesth 2011;25:975-80.

25. McEwen BS. Neural gonadal steroid actions. Science 1981;211:1303-11.

26. Silva NL, Boulant JA. Effects of testosterone, estradiol, and temperature on neurons in preoptic tissue slices. Am J Physiol 1986;250:R625-32.

27. Neal JL, Lamp JM, Lowe NK, et al. Differences in inflammatory markers between nulliparous women admitted to hospitals in preactive vs active labor. Am J Obstet Gynecol 2015;212:68.e1-8.

28. Lange EMS, Segal S, Pancaro C, et al. Association between intrapartum magnesium administration and the incidence of maternal fever: a retrospective cross-sectional study. Anesthesiology 2017;127:942-52.

29. Curtin WM, Katzman PJ, Florescue H, et al. Intrapartum fever, epidural analgesia and histologic chorioamnionitis. J Perinatol 2015;35:396-400.

30. Acker DB, Johnson MP, Sachs BP, et al. The leukocyte count in labor. Am J Obstet Gynecol 1985;153:737-9. 
Supplementary

Table S1 Characteristics of study population according to epidural analgesia administration

\begin{tabular}{lccc}
\hline Variables & No epidural analgesia $(\mathrm{n}=17,610)$ & Epidural analgesia $(\mathrm{n}=18,952)$ & $\mathrm{P}$ value \\
\hline Maternal age $\geq 35$ years & $891(5.1)$ & $1,165(6.1)$ & $<0.001$ \\
$\mathrm{BMI} \geq 25 \mathrm{~kg} / \mathrm{m}^{2}$ & $11,199(63.6)$ & $12,111(63.9)$ & 0.542 \\
Labor length $>600 \mathrm{~min}$ & $1,438(8.2)$ & $5,375(28.4)$ & $<0.001$ \\
Birth weight $>4.0 \mathrm{~kg}$ & $464(2.6)$ & $739(3.9)$ & $<0.001$ \\
WBC & $8.6(7.3-10.2)$ & $8.5(7.2-10.0)$ & $<0.001$ \\
Neutrophil baseline $\left(10^{9} / \mathrm{L}\right)$ & $6.27(5.09-7.77)$ & $6.21(5.04-7.60)$ & $<0.001$ \\
Monocyte baseline $\left(10^{9} / \mathrm{L}\right)$ & $0.58(0.47-0.72)$ & $0.58(0.47-0.71)$ & 0.104 \\
Lymphocyte baseline $\left(10^{9} / \mathrm{L}\right)$ & $1.57(1.30-1.89)$ & $1.56(1.31-1.87)$ & 0.249 \\
Lymphocyte count $\leq 1.1 \times 10^{9} / \mathrm{L}$ & $1,815(9.6)$ & $1,790(10.2)$ & 0.060 \\
Lymphocyte/neutrophil & $0.25(0.19-0.33)$ & $0.25(0.19-0.33)$ & 0.028 \\
Fever & $554(3.1)$ & $2,714(14.3)$ & $<0.001$
\end{tabular}

Continuous variables were presented as median (IQR); counting variables were presented as number (percentage). BMI, body mass index; WBC, white blood cell; IQR, interquartile range. 